\title{
LEFT AND RIGHT INVERSES IN GROUP ALGEBRAS
}

\author{
BY M. SUSAN MONTGOMERY
}

Communicated by I. N. Herstein, January 17, 1969

If $G$ is a group, denote the discrete group algebra over the complex numbers by $C[G]$ and the $n \times n$ matrices over $C[G]$ by $C[G]_{n}$. I. Kaplansky has observed [2] that any element of $C[G]_{n}$ which is right invertible is also left invertible (for an application of this result, see [1]). Kaplansky's observation followed from known results in the theory of rings of operators concerning von Neumann algebras. This note presents a proof using instead the uniform closure of $C[G]$, which is a $C^{*}$-algebra, and very elementary properties of $C^{*}$-algebras. Kaplansky himself in several lectures has indicated that the result could be obtained by using the uniform closure rather than the weak closure of $C[G]$. Since the result does present itself in the works of others and appears to be needed, it seems worthwhile to put it offcially in the literature. It would be interesting to try to find a purely algebraic proof of the result, especially for any field, since the result for fields of characteristic $p$ is as yet unknown. The proof is only given for $C[G]$ itself, but a similar argument will work for $C[G]_{n}, n>1$; since $C[G]_{n} \cong C_{n}[G]$, one may use the trace of the matrix which is the coefficient of the identity.

For any element $x$ of $C[G]$, write $x=\sum_{g \in G} \alpha_{g} g$, where $\alpha_{g} \in C$ and $\alpha_{o}=0$ for all but a finite number of $g \in G$. C [G] has an involution *, given by $x^{*}=\sum_{g \in G} \bar{\alpha}_{g} g^{-1}$. One may also define a trace $t$ on $C[G]$ by $t(x)=\alpha_{1}$, where $\alpha_{1}$ is the coefficient of $1 \in G$. $t$ has the following properties:

(1) $t(1)=1$.

(2) $t$ is $C$-linear.

(3) $t(x y)=t(y x)$, all $x, y$ in $C[G]$.

(4) $t\left(x x^{*}\right) \geqq 0$, and $t\left(x x^{*}\right)=0 \Rightarrow x=0$.

$C[G]$ has an inner product given by $\left(\sum \alpha_{\theta} g, \sum \beta_{g} g\right)=\sum \alpha_{\theta} \bar{\beta}_{\theta}$, and thus may be completed to a Hilbert space $H$, with norm \|\|$_{0}$. Note that if $x=\sum \alpha_{g} g \in C[G]$, then $\|x\|_{0}=\left(\sum\left|\alpha_{g}\right|^{2}\right)^{1 / 2}=t\left(x x^{*}\right)^{1 / 2}$. For any $x$ in $C[G]$, left multiplication by $x$ (denoted $L_{x}$ ) is an element of $B(H)$, the bounded linear operators on $H$. Let \|\| denote the operator norm in $B(H)$; then $\left\|L_{x}\right\| \geqq\|x\|_{0}$, and if $g \in G$ then $L_{0}$ is unitary (as $\|g x\|_{0}$ $=\|x\|_{0}$, for all $x \in C[G]$ ). Also, $L_{x}^{*}=L_{x^{*}}$, where $L_{x}^{*}$ is the operator adjoint to $L_{x}$.

Let $\mathscr{A}$ be the closure of $\left\{L_{x}\right\}_{x \in \mathrm{C}[G]}$ with respect to \|\| in $B(H)$. $\mathscr{A}$ is a $C^{*}$-algebra, and so $1+A A^{*}$ is invertible for all $A \in \Re$. By a purely 
formal argument, it then follows that every idempotent in $\mathfrak{A}$ is similar to a projection [3].

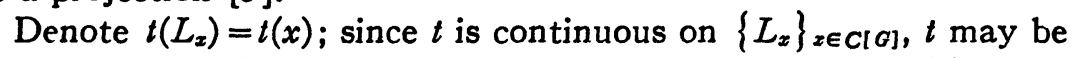
extended to all of $\mathfrak{A}$. $t$ clearly satisfies properties (1), (2) and (3) above on $\mathfrak{A}$; only a proof of (4) is required.

Lemma 1. If $A \in \mathcal{P}$ and $t\left(A A^{*}\right)=0$, then $A=0$.

Proof. Choose $x_{n} \in C[G]$ so $\lim _{n \rightarrow \infty}\left\|L_{x_{n}}-A\right\|=0$. Then

$$
\lim _{n \rightarrow \infty}\left\|L_{x_{n}} L_{x_{n}}^{*}-A A^{*}\right\|=0 \text {, }
$$

so

$$
\lim _{n \rightarrow \infty}\left\|x_{n}\right\|_{0}^{2}=\lim t\left(x_{n} x_{n}^{*}\right)=\lim t\left(L_{x_{n}} L_{x_{n}}^{*}\right)=t\left(A A^{*}\right)=0 .
$$

To show $A=0$, it will suffice to show $A g=0$, all $g \in G$ (since every element of $C[G]$ is a finite linear combination of the $g$ 's, and $C[G]$ is dense in $H$ )

$$
\begin{array}{r}
\|A g\|_{0} \leqq\left\|x_{n} g-A g\right\|_{0}+\left\|x_{n} g\right\|_{0} \leqq\left\|L_{x_{n}}-A\right\|\|g\|_{0}+\left\|x_{n} g\right\|_{0} \\
=\left\|L_{x_{n}}-A\right\|+\left\|x_{n}\right\|_{0} .
\end{array}
$$

LEMMA 2. Let e be any idempotent in $C[G]$. Then $0 \leqq t(e) \leqq 1$, and $t(e)=0 \Rightarrow e=0, t(e)=1 \Rightarrow e=1$.

Proof. $L_{0}$ is an idempotent in $\mathscr{A}$, so $L_{0}=A^{-1} P A$, where $P$ is a projection. $t(P)=t(e)$ by Property (3). Now $P=P P^{*}$, so $t(P)>0$ if $P \neq 0$ by Lemma 1. Similarly $t(1-P)>0$ if $P \neq 1$, and thus $1>t(P)>0$.

TheOREM. If $x$ and $y$ are in $C[G]$ and $x y=1$, then $y x=1$.

Proof. Since $x y=1, y x$ is an idempotent. $t(y x)=t(x y)=1$, so $y x=1$ by Lemma 2 .

\section{REFERENCES}

1. W. H. Cockcroft and R. G. Swan, On the homotopy type of certain two-dimensional complexes, Proc. London Math. Soc. (3) 11 (1961), 194-202.

2. I. Kaplansky, Fields and rings, Univ. of Chicago Press, Chicago, III., 1969, p. 122.

3. - Rings of operators, Benjamin, New York, 1968, pp. 24, 34.

University of Chicago, Chicago, Illinois 60637 